\title{
The Construction of the Non-Material Incentive Model for the Firm Operator's Compensation Contract in China
}

\author{
Biao Luo ${ }^{1}$, Shan-Shan Zheng ${ }^{1}$, Hong-Mei Ji ${ }^{2}$, Chao Gong ${ }^{1}$ \\ ${ }^{1}$ School of Management, University of Science and Technology of China, Hefei, China; ${ }^{2}$ School of Business, Anhui University, He- \\ fei, China. \\ Email: ustcji@163.com
}

Received June $27^{\text {th }}, 2012$; revised July $26^{\text {th }}, 2012$; accepted August $10^{\text {th }}, 2012$

\begin{abstract}
Effective compensation contract is the most driving force that encourages the operator of the firm to work hard. This article, considering the ability of the operator, built a model of compensation contract with some explanatory variables, such as effort level and risk attitude of the operator, coefficient of input flexibility, and coefficient of discount. Then, it tried to use the principal-agent theory to solve the model of explicit function, and analyzed the model based on material incentive and non-material incentive in the mechanism of profit sharing; the conclusion had important practical significance for the firm to implement the performance management.
\end{abstract}

Keywords: Compensation Contract; Material Incentive; Non-Material Incentive; Incentive Mechanism

\section{Introduction}

Over the past decades, incentive of the modern firm is one of the most prevalent problems which are widely concerned and focused by academic and business circles. Perfect compensation contract can enhance the cohesiveness of the firm and lead the operator do its best according to the owner expected direction, which can bring more profits to the firm. The academic literature on agency theory and executive compensation has argued that operator compensation should be aligned to firm performance [1-3] (see for example, Holmstrom, 1979; Grossman and Hart, 1983; and Jensen and Murphy, 1990). Many scholars take combined incentives for breakthrough point, which research angle includes but not limited to short-long term combine incentives [4] (see for example, Zhang Yong, 2004), and explicit-implicit combine incentives [5] (see for example, Chen Shuang-ying $\&$ Tang Xiao-wo, 2005), etc.

Firms can provide value in return for their operator of four aspects: 1) compensation: base salary, bonuses, operator stock ownership and stock options; 2) Learning and improving: training, career design, performance management; 3) working environment: organizational climate, leadership, performance support, life and work balance; 4) benefits: health, insurance, operator assistance programs, savings, vacation. Among them, the nonmonetary forms of payment to operator and the ability to affect or change the work of the operator and the results of the work so that operator make a greater contribution to organizational goals of factors, known as non-material incentives [6]. However, academic and business circles paid more attention to material incentive. Non-material incentive is always set on the second position no matter for theory or practice, which not only affects the completeness of incentive mechanism and also weakens the result of material incentive. Thus, material incentives to achieve balanced and non-material incentives [7] (Chen Shuang-ying \& Tang Xiao-wo et al., 2005)

This paper extended the model by deriving the optimal division of operator compensation into material and nonmaterial, constructed the explicit function model of operator compensation contract, quantified the material incentive, and analyzed the micro relation between nonmaterial incentive and explanation variables to get the way to increase owner net earnings under the material and non-material incentive combination situation in theory. The paper is organized as follows. In the following section, we summarize the non-material incentive factors. Section 3 presents the assumption and model. Section 4 analyzes and discusses the results. Section 5 concludes.

\section{Literature Review}

Incentives are increasingly popular in the corporate sector, where operator considers innovative human resource practice, such as awards, to be essential for firm competitiveness [8] (Frey, 2007). Nelson (2005), for example, provides ample evidence of the number and variety of incentives for companies. The prevalence and popularity 
of incentives in the corporate sector suggests that incentives full of important functions in leading agent to take action in accordance with the principal goal. The theory of agency deems that the way to make the agent to take the best behavior is pay for performance. Interestingly, most of compensation research has so far limited to material incentive, Such as awards (Frey, 2007), ownership incentive [9] (Jenson \& Meckling, 1976), income incentive (including salary and bonus, etc.) and stock-based compensation [10] (Jenson \& Murphy, 1981; Stephen Bryan, 2000; Todd T. Milbourn, 2003). It is well known that human need is multilevel and multiple perspectives in Maslow's hierarchy of needs. This opinion is also described by ERG theory, what said people can have more than one need, and plus all levels of needs are less being satisfied, the more being longed for by people. However, recent research on incentive systems (Prendergast, 1999; Daily et al., 2003) suggests that material incentive does not always work perfectly. This holds, in particular, when the task to be performed is difficult, or impossible, to specify ex-ante, or to monitor ex-post. In this case, it is hard to make a monetary payment which is considered to be fair by the recipients. "Soft" awards or can be said non-material incentive (e.g. Holmstrom \& Milgrom, 1991, 1994), which endeavor to take a broader view of the agent's efforts, become more useful then. Such incentive is splendidly suited to that purpose. With a few exceptions in the military branch orders, decorations and prizes are given to honor more conventional efforts, rather than very specific efforts.

As said above, non-material can save the incentive cost and restrict operator behaviors under certain conditions. Many research scholars had taken non-material incentive to be the part of the operator's compensation, and reflect it into their contracts model. Bryan et al. (2000) thought stock-based compensation for the operator. RÄudiger Fahlenbrach (2003) had taken shareholder rights into operator compensation contract. Li Yuan et al. (2002) focused on the measuring model of non-material incentive, and build the optimal model of the combination incentive to operator [11]. On the base of the static analysis, he found that operator utility can be improved under the restraints of certainty material condition and incentive cost. He Jia-tie et al. (2005) constructed the incentive combination model based on the implicit value and unqualified demand of top operator based on analyzing implicit value and implicit demand of top operator to overcome the limitation of traditional single monetary incentive [12]. However, all of these studies are using implicit function to form the non-material incentive, and can't analyze the influence of explanatory variables to the key model parameters accordingly. Liu Gui-liang et al. [13] (2007), Zhang Jian et al. [14] (2010) although built compensation contract with explicit function; they both overlooked the costs from the non-material incentive.

Obviously, this is not match the realities that owner carry out non-material incentive is not free, and also the income brought to operator and costs brought to the owner is not equal. For that reason, this paper is to research the incentive problem of operator in firm; we choose the material motivation factor and non-material motivation factor to synthetically reckon with. In addition, different from their research, this paper does not take the ability level of operator as an exogenous variable any more, but rather regard as an important explanatory variable taking into the model, and analyses the influence to firm performance and operator effort level. Therefore, we should incorporate the material motivation and nonmaterial motivation to motivate operator which can minimize the incentive cost, and also maximum satisfy the organization's expectations and individual wish.

\section{The Basic Model}

\subsection{Fundamental Assumption}

Proposition 1. If the firm performance function is linear, effort has little effect in large firms. Thus it is optimal to implement an interior level of effort, to avoid exerting excessive costs on the operator. So we assume that the function of firm performance outputs is linear, which can be written as follows:

$$
\pi=h n+\theta,
$$

where $\pi$ is the outputs of firm performance, $h$ is effort level of operator, $n$ is ability level of operator, $\theta$ is an is an endogenous variable, $\theta \sim N\left(0, \sigma^{2}\right)$.

Proposition 2. We assume owner is risk neutral, operator is risk aversion. The utility function of operator is constantly absolute risk averse, which the function form is :

$$
E u=-E e^{-\rho \omega}=-e^{-\rho\left\{E \omega-\frac{1}{2} \rho \operatorname{var}(\omega)\right\}},
$$

where $\rho$ is a constant absolute risk aversion variable that satisfies the condition $\rho \succ 0$, and $\omega$ is actual income of operator.

Proposition 3. We assume the function of operator effort cost can be written as follows:

$$
f(h, n)=\frac{b h^{2}}{2 n},
$$

where $b$ is the effort cost coefficient. The bigger $b$ is, the more disutility taken by the same effort level $h$. In other words, the bigger $n$ is, the little disutility taken by the same effort level $h$ for operator had different ability levels.

Proposition 4. Owner takes material incentive measures and non-material incentive measures simultaneously. 
In our setting, the cost of incentives is a function of the operator's wage, but the benefits of effort are a function of firm value, which is substantially greater. Because maximum effort is always optimal, the efficient contract takes a simple form. Since effort has a percentage effect on both firm value and utility, the percentage change in pay for a percentage firm return is the relevant incentive measure, and it must be sufficiently high to induce maximum effort. Translated into real variables, this measure equals the proportion of total salary that is comprised of material and non-material. Thus, the fraction of pay that must be composed of non-material should be constant across operator of different salaries. We assume that the cost of non-material incentive measures is $\lambda \gamma \pi$, where current non-material incentive measures can make the next issue income of operator increase by $\gamma \pi$, which $\lambda$ is the elastic coefficient of the non-material incentive inputs, said the material incentive effect of input and output. If the condition $0 \leq \lambda \leq 1$ holds, the owner will simply adopt the material incentive measures which meet condition that the perceived equivalent income by the operator is equal or greater than the monetary inputs of non-material incentive. The function of operator compensation contract is as follows:

$$
\mathrm{S}(\pi)=\alpha+\beta \pi+\varepsilon \gamma \pi,
$$

where $\alpha$ is operator fixed income, $\beta$ is coefficient of share profits which reflect the operator share degree of outputs, $\varepsilon$ is discount coefficient, and $\varepsilon \gamma \pi$ is the increased monetary of operator current income by the discounted non-material incentive.

\subsection{Model Formulation and Solution Procedure}

Based on above fundamental assumptions, we get the utility function of firm performance outputs as follows:

$$
\begin{gathered}
E \pi=E(h n+\theta)=h n, \\
\operatorname{var}(\pi)=\sigma^{2} .
\end{gathered}
$$

The expected utility function of owner is equal to its net earnings because he is risk neutral, which can be written as follows:

$$
E[\pi-\alpha-\beta \pi-\lambda \gamma \pi]=(1-\beta-\lambda \gamma) h n-\alpha,
$$

The actual income function of operator is operator is risk aversion neutral in considering efforts costs. Specifically, we use the functional form:

$$
\begin{gathered}
\omega=S(\pi)-f(h, n)=\alpha+\beta \pi+\varepsilon \gamma \pi-\frac{b h^{2}}{2 n}, \\
E \omega=\alpha+(\beta+\varepsilon \gamma) h n-\frac{b h^{2}}{2 n} .
\end{gathered}
$$

In the meanwhile, operators have to take risks, the ac- tual income satisfy the condition:

$$
\operatorname{var}(\omega)=(\beta+\varepsilon \gamma)^{2} \sigma^{2} .
$$

Consequently, Agent Certainty Equivalence (ACE) can be written as follows:

$$
\begin{aligned}
\mathrm{ACE} & =E \omega-\frac{1}{2} \rho \operatorname{var}(\omega) \\
& =\alpha+(\beta+\varepsilon \gamma) h n-\frac{b h^{2}}{2 n}-\frac{1}{2} \rho(\beta+\varepsilon \gamma)^{2} \sigma^{2},
\end{aligned}
$$

if $\omega_{0}$ is operator reservation utility, then participation constraint is

$$
\text { IR : } \mathrm{ACE} \geq \omega_{0},
$$

the formula get an equal sign in the optimal principle. Using the first-order conditions, we obtain the operator's incentive compatibility constraint as

$$
\frac{\partial \mathrm{ACE}}{\partial h}=(\beta+\varepsilon \gamma) n-\frac{b h}{n}=0,
$$

which also can be written as

$$
\mathrm{IC}: h^{*}=\frac{(\beta+\varepsilon \gamma) n^{2}}{b} .
$$

From the above equation, we can see $h^{*}$ increases with $n$, which means the bigger operator ability is, the higher its optimal effort level is.

Therefore, the compensation contract can be described as

$$
\max :(1-\beta-\lambda \gamma) h n-\alpha
$$

S.T.

$$
\begin{aligned}
& \text { IR }: \text { ACE } \geq \omega_{0} \\
& \mathrm{IC}: h^{*}=\frac{(\beta+\varepsilon \gamma) n^{2}}{b} \\
& 0 \leq \beta, \gamma, \lambda, \varepsilon \leq 1, \alpha \leq \omega_{0}
\end{aligned}
$$

and substituting the constraint condition into the target function $\max$, we get $\max : f(\alpha, \beta)$ :

$\max$ :

$$
\begin{aligned}
f(\alpha, \beta)= & \frac{(\beta+\varepsilon \gamma)[1+(\varepsilon-\lambda) \gamma] n^{3}}{b} \\
& -\frac{(\beta+\varepsilon \gamma)^{2}\left(n^{3}+\rho b \sigma^{2}\right)}{2 b}-\omega_{0}
\end{aligned}
$$

where $\alpha$ is operator fixed income, $\beta$ is coefficient of share profits which reflect the operator share degree of outputs, $n$ is ability level of operator, $\lambda$ is the elastic coefficient of the non-material incentive inputs, said the material incentive effect of input and output, $\varepsilon$ is discount coefficient, $\rho$ is a constant absolute risk aversion variable that satisfies the condition $\rho \succ 0, b$ is the 
effort cost coefficient, $h$ is effort level of operator, $\gamma$, $\sigma$ are constant variables.

We generally follow the solution procedures:

First, a calculating the first derivative of the Lagrange function (1), so as to get the first-order condition of $f(\alpha, \beta)$ respect to $\beta$ as Equation (2),

$\frac{\partial f(\alpha, \beta)}{\partial \beta}=\frac{[1+(\varepsilon-\lambda) \gamma] n^{3}}{b}-\frac{(\beta+\varepsilon \gamma)\left(n^{3}+\rho b \sigma^{2}\right)}{b}$

The optimality condition then is given by Equation (3)

$$
\frac{\partial f(\alpha, \beta)}{\partial \beta}=0
$$

w.r.t. Equation (3), we get

$$
\frac{[1+(\varepsilon-\lambda) \gamma] n^{3}}{b}-\frac{(\beta+\varepsilon \gamma)\left(n^{3}+\rho b \sigma^{2}\right)}{b}=0
$$

simplify the Equation (4), we get

$$
(\beta+\varepsilon \gamma)\left(n^{3}+\rho b \sigma^{2}\right)-[1+(\varepsilon-\lambda) \gamma] n^{3}=0
$$

w.r.t. $\beta$, and solving for $\beta^{*}=\beta$, we get

$$
\beta^{*}=\beta=\frac{n^{3}-\left(\lambda n^{3}+\varepsilon \rho b \sigma^{2}\right) \gamma}{n^{3}+\rho b \sigma^{2}}
$$

Second, substituting $\beta^{*}$ into participation constraint, when the condition IR: $\mathrm{ACE} \geq \omega_{0}$ takes the equal sign, we get

$$
\begin{aligned}
\mathrm{ACE} & =E \omega-\frac{1}{2} \rho \operatorname{var}(\omega) \\
& =\alpha+(\beta+\varepsilon \gamma) h n-\frac{b h^{2}}{2 n}-\frac{1}{2} \rho(\beta+\varepsilon \gamma)^{2} \sigma^{2} \\
& =\omega_{0}
\end{aligned}
$$

where $h=\frac{(\beta+\varepsilon \gamma) n^{2}}{b}$,

w.r.t. $\alpha^{*}$, and solving for $\alpha^{*}=\alpha$, we get

$$
\alpha^{*}=\omega_{0}-\frac{n^{6}[1+(\varepsilon-\lambda) \gamma]^{2}\left(n^{3}-\rho b \sigma^{2}\right)}{2 b\left(n^{3}+\rho b \sigma^{2}\right)^{2}}
$$

Finally, substituting $\alpha^{*}$ and $\beta^{*}$ into the target function $\max$ in formula $\left({ }^{*}\right)$, we get:

$$
\begin{aligned}
f\left(\alpha^{*}, \beta^{*}\right) & =\left(1-\beta^{*}-\lambda \gamma\right) \mathrm{hn}-\alpha^{*} \\
& =\frac{n^{6}[1+(\varepsilon-\lambda) \gamma]^{2}}{2 b\left(n^{3}+\rho b \sigma^{2}\right)}-\omega_{0}
\end{aligned}
$$

\subsection{Results}

We can get the first-order results of Equation (6) respect to $\beta, \varepsilon, n, b, \rho$ as follows:

$$
\begin{aligned}
& \frac{\partial \beta^{*}}{\partial \lambda}=\frac{\partial\left[\frac{n^{3}-\left(\lambda n^{3}+\varepsilon \rho b \sigma^{2}\right) \gamma}{n^{3}+\rho b \sigma^{2}}\right]}{\partial \lambda}=-\frac{\gamma n^{3}}{n^{3}+\rho b \sigma^{2}} \prec 0, \\
& \frac{\partial \beta^{*}}{\partial \varepsilon}=\frac{\partial\left[\frac{n^{3}-\left(\lambda n^{3}+\varepsilon \rho b \sigma^{2}\right) \gamma}{n^{3}+\rho b \sigma^{2}}\right]}{\partial \varepsilon}=-\frac{\gamma \rho b \sigma^{2}}{n^{3}+\rho b \sigma^{2}} \prec 0,
\end{aligned}
$$

$$
\begin{aligned}
& \frac{\partial \beta^{*}}{\partial n} \\
& =\frac{\partial\left[\frac{n^{3}-\left(\lambda n^{3}+\varepsilon \rho b \sigma^{2}\right) \gamma}{n^{3}+\rho b \sigma^{2}}\right]}{\partial n} \\
& =\frac{3 n^{2}(1-\lambda \gamma)\left(n^{3}+\rho b \sigma^{2}\right)-\left[n^{3}-\left(\lambda n^{3}+\varepsilon \rho b \sigma^{2}\right) \gamma\right] 3 n^{2}}{\left(n^{3}+\rho b \sigma^{2}\right)^{2}}, \\
& =\frac{3 \rho b n^{2} \sigma^{2}[1+\gamma(\lambda-\varepsilon)]}{\left(n^{3}+\rho b \sigma^{2}\right)^{2}}
\end{aligned}
$$

since the condition $0 \leq \gamma, \lambda, \varepsilon \leq 1$ holds, the following inequality holds,

$$
\begin{gathered}
-1 \leq \gamma(\lambda-\varepsilon) \leq 1, \\
1+\gamma(\lambda-\varepsilon) \succ 0, \\
\frac{\partial \beta^{*}}{\partial n} \succ 0 .
\end{gathered}
$$

$\frac{\partial \beta^{*}}{\partial \rho}$

$=\frac{\partial\left[\frac{n^{3}-\left(\lambda n^{3}+\varepsilon \rho b \sigma^{2}\right) \gamma}{n^{3}+\rho b \sigma^{2}}\right]}{\partial \rho}$

$$
=\frac{-\gamma \varepsilon b \sigma^{2}\left(n^{3}+\rho b \sigma^{2}\right)-\left[n^{3}-\left(\lambda n^{3}+\varepsilon \rho b \sigma^{2}\right) \gamma\right] b \sigma^{2}}{\left(n^{3}+\rho b \sigma^{2}\right)^{2}}
$$

$$
=\frac{b n^{3} \sigma^{2}[\gamma(\lambda-\varepsilon)-1]}{\left(n^{3}+\rho b \sigma^{2}\right)^{2}}
$$

since the condition $0 \leq \gamma, \lambda, \varepsilon \leq 1$ holds, the following inequality holds,

$$
\begin{gathered}
-1 \leq \gamma(\lambda-\varepsilon) \leq 1, \\
\gamma(\lambda-\varepsilon)-1 \prec 0, \\
\frac{\partial \beta^{*}}{\partial \rho} \prec 0 .
\end{gathered}
$$




$$
\begin{aligned}
& \frac{\partial \beta^{*}}{\partial b} \\
& =\frac{\partial\left[\frac{n^{3}-\left(\lambda n^{3}+\varepsilon \rho b \sigma^{2}\right) \gamma}{n^{3}+\rho b \sigma^{2}}\right]}{\partial b} \\
& =\frac{-\gamma \varepsilon \rho \sigma^{2}\left(n^{3}+\rho b \sigma^{2}\right)-\left[n^{3}-\left(\lambda n^{3}+\varepsilon \rho b \sigma^{2}\right) \gamma\right] \rho \sigma^{2}}{\left(n^{3}+\rho b \sigma^{2}\right)^{2}} \\
& =\frac{\rho n^{3} \sigma^{2}[\gamma(\lambda-\varepsilon)-1]}{\left(n^{3}+\rho b \sigma^{2}\right)^{2}}
\end{aligned}
$$

since the condition $0 \leq \gamma, \lambda, \varepsilon \leq 1$ holds, the following inequality holds,

$$
\begin{gathered}
-1 \leq \gamma(\lambda-\varepsilon) \leq 1, \\
\gamma(\lambda-\varepsilon)-1 \prec 0, \\
\frac{\partial \beta^{*}}{\partial b} \prec 0 .
\end{gathered}
$$

Other first-order results of Equations (8) and (9) respect to $\beta, \varepsilon, n, b, \rho$ will be seen in appendix.

\section{Numerical Analysis and Discussion}

\subsection{Influence of Factors to Coefficient of Sharing Profits}

We can get from formula (6) as follows:

1) Input elasticity coefficients: $\frac{\partial \beta^{*}}{\partial \gamma} \prec 0$, which means $\beta^{*}$ increases with the decrease of $\lambda$. Put another way, the smaller the input elasticity coefficients is, the bigger utility of non-material incentives to operator, that is to say the owner should set a larger coefficient of sharing profits to operator.

2) Discount coefficient: $\frac{\partial \beta^{*}}{\partial \varepsilon} \prec 0$, which means $\beta^{*}$ increases with the decrease of $\varepsilon$. If the discount coefficient is smaller, indicates that the adverse impact of the future environment on the income is larger, the owner should set a larger coefficient of sharing profits to operator too.

3) Ability level: $\frac{\partial \beta^{*}}{\partial n} \succ 0$, which means $\beta^{*}$ increases with the increase of $n$. The result indicates that if the operator's ability is higher, the owner should set a larger coefficient of sharing profits to operator. All other things being equal, operator who had a higher ability appear willing to take more risk.

4) Degree of risk aversion: $\frac{\partial \beta^{*}}{\partial \rho} \prec 0$, which means $\beta^{*}$ decreases with the increase of $\rho$. If the degree of operator's risk aversion is higher, the owner should set a less coefficient of sharing profits.

5) Effort cost coefficient: $\frac{\partial \beta^{*}}{\partial \mathrm{b}} \prec 0$, which means $\beta^{*}$ decreases with the increase of $b$. The result demonstrates that the bigger operator effort cost coefficient is, the less the coefficient of sharing profits is.

\subsection{Influence of Factors to Fixed Wage}

We can get from formula (8) as follows:

1) Input elasticity coefficients: $\frac{\partial \alpha^{*}}{\partial \lambda} \succ 0$, which means $\alpha^{*}$ increases with the decrease of $\lambda$. The result states clearly that the less utility of non-material incentives to operator, the high level of fixed wage should owner set.

2) Discount coefficient: $\frac{\partial \alpha^{*}}{\partial \varepsilon} \prec 0$, which means $\alpha^{*}$ increases with the decrease of $\varepsilon$. A smaller discount coefficient indicates that the adverse impact of the future environment on the income is larger; the owner should set a high level of fixed wage.

3) Ability level: $\frac{\partial \alpha^{*}}{\partial n} \prec 0$, which means $\alpha^{*}$ increases with the increase of $n$. The result indicates that if the operator's ability is higher, the owner should set a lower level of fixed wage to operator.

4) Degree of risk aversion: $\frac{\partial \alpha^{*}}{\partial \rho} \succ 0$, which means $\alpha^{*}$ increases with the increase of $\rho$. If the degree of operator's risk aversion is higher, the owner should set a lower level of fixed wage to operator.

5) Effort cost coefficient: $\frac{\partial \alpha^{*}}{\partial b} \succ 0$, which means $\alpha^{*}$ increases with the increase of $b$. The result demonstrates that the bigger operator effort cost coefficient is, the higher level of fixed wage is.

\subsection{Influence of Factors to Owner Net Earnings}

We can get from formula (9) as follows:

1) Input elasticity coefficients: $\frac{\partial f\left(\alpha^{*}, \beta^{*}\right)}{\partial(\lambda)} \prec 0$, which means $f\left(\alpha^{*}, \beta^{*}\right)$ increases with the decrease of $\lambda$. The result states clearly that the more utility of non-material incentives to operator, the high owner net earnings is.

2) Discount coefficient: $\frac{\partial f\left(\alpha^{*}, \beta^{*}\right)}{\partial \varepsilon} \succ 0$, which means $f\left(\alpha^{*}, \beta^{*}\right)$ increases with the increase of $\varepsilon$. A smaller 
discount coefficient indicates that the adverse impact of the future environment on the income is smaller; the owner net earnings should be larger.

3) Ability level: $\frac{\partial f\left(\alpha^{*}, \beta^{*}\right)}{\partial n} \succ 0$, which means

$f\left(\alpha^{*}, \beta^{*}\right)$ increases with the increase of $n$. The result indicates that if the operator's ability is higher, the owner net earning should be larger.

4) Degree of risk aversion: $\frac{\partial f\left(\alpha^{*}, \beta^{*}\right)}{\partial \rho} \prec 0$, which means $f\left(\alpha^{*}, \beta^{*}\right)$ increases with the decrease of $\rho$. If the degree of operator's risk aversion is lower, the owner net earning should be larger.

5) Effort cost coefficient: $\frac{\partial f\left(\alpha^{*}, \beta^{*}\right)}{\partial(\lambda)} \prec 0$, which means $f\left(\alpha^{*}, \beta^{*}\right)$ increases with the decrease of $b$. The result demonstrates that the smaller operator's effort cost coefficient is, the larger the owner net earning is.

\subsection{Non-Material Incentives and Total Incentive Costs}

In the process of building and solving the model, we can get: if non-material incentives are adopting by organization, the indirect cost is $\lambda \gamma \pi$ in the current period, and operator increased income is $\gamma \pi$ the next period. In that situation, the non-material incentive with the character of saving cost (when the condition $0 \leq \lambda \leq 1$ holds) is not the optimal incentive in the long run. The degree of saving cost is influenced by the ability of guaranteeing income cashed in of the future environment. Only in the case that the discount coefficient is bigger than elastic coefficient (namely $\varepsilon-\lambda \succ 0$ ), the non-material incentive can really save costs for the owner, maximize the utility of operator in the certain total incentive costs or minimize the total incentive cost under the condition of the unchanged operator utility.

A bigger discount coefficient which means the adverse effect of future environment to income is smaller, can bring a bigger incentive utility to an operator with nonmaterial incentive from the formula (5). Aiming to reduce costs of material incentive and total incentive, owner tends to substitute material incentive with efficient non-material under the condition that the agent certain equivalent is greater than or equal to the revenue reserves. Of course, this substitution effect is limited, since it has the boundary decreasing effect. Additionally, non-material incentive may bring new problems, such as increasing the human resource cost in the long run. For this reason, owner may take the non-material incentive measures to the older the operator.

\section{Conclusions}

This article studies operator compensation contract based on the combination of material and non-material incentive, expands the principal-agent theory, provides an important theoretical basis for the optimal design of the compensation contract. The conclusion had an important practical significance for the firm to implement the performance management. Therefore, we can get conclusions as follows:

First, the higher ability level, the less degree of risk aversion, the more utility of non-material and the adverse effect of future environment to income are. The owner should set a higher coefficient of sharing profits. Second, if the owner takes the combination of material and nonmaterial incentive mode, a lower elastic coefficient or a higher discount coefficient indicates that the owner should set a lower level of fixed wages to operator. Finally, we can improve the owner earnings through the following ways at least: The firm can bring forward the appropriate non-material incentive ways after analyzing the multi-level demands of operator to meet the condition that elastic coefficient of the non-material incentive is less than the discount coefficient; the firm should make the operator better understand future income environment through providing comprehensive, multi-channel information, and make them feel the discount coefficient is close to the real discount coefficient; the firm can improve the operator ability level through diversity training and opportunities; the firm can encourage operator learning risk management knowledge, and to reduce the operator degree of risk aversion.

\section{Acknowledgements}

This paper is founded by the youth science fund projects of the national natural science foundation, No. 70802058, and the national innovation research group projects of national natural science foundation, No. 70821001, and the fifth Science and Technological Fund of Anhui Province for Outstanding Youth, NO: 10040606Y35.

\section{REFERENCES}

[1] B. Hölmstrom, "Moral Hazard and Observability," The Bell Journal of Economics, Vol. 10, No. 1, 1979, pp. 7491. doi: $10.2307 / 3003320$

[2] S. J. Grossman and O. D. Hart, "An Analysis of the Principal-Agent Problem," Econometrica, Vol. 51, No. 1, 1983, pp. 7-45. doi: $10.2307 / 1912246$

[3] M. Jensen and K. Murphy, "Performance Pay and TopManagement Incentives," Journal of Political Economy, Vol. 98, No. 2, 1990, pp. 225-264.

[4] Y. Zhang, "Study Excellent Incentive Combination of Operator: Long-Term and Short-term Reward," Journal of Industrial Engineering and Engineering Management, 
Vol. 18, No. 3, 2004, pp. 125-127.

[5] S.-Y. Chen, X.-W. Tang, D.-B. Ni and Y.-K. Ma, "Value Analysis on Non-Material Incentive in Combination Incentive to Operator," Chinese Journal of Management Science, Vol. 13, No. 1, 2005, pp. 122-126.

[6] M. Kosfeld and S. Neckermann, "Getting More Work for Nothing? Symbolic Awards and Worker Performance," American Economic Journal: Microeconomics, Vol. 3, No. 3, 2011, pp. 86-99. doi:10.1257/mic.3.3.86

[7] S.-Y. Chen and X.-W. Tang, "Studies on Combination Contract of Explicit and Implicit Incentives to Human Capital," Chinese Journal of Management Science, Vol. 13, No. 6, 2005, pp. 108-112.

[8] B. S. Frey, "Awards as Compensation," European Management Review, Vol. 4, No. 1, 2007, pp. 6-14. doi:10.1057/palgrave.emr.1500068

[9] M. Jensen and W. Meckling, "Theory of the Firm: Operational Behavior, Agency Costs and Ownership Structure," Journal of Financial Economics, Vol. 3, No. 4, 1976, pp. 305-360. doi:10.1016/0304-405X(76)90026-X
[10] T. T. Milbourn, "Operator Reputation and Stock-Based Compensation," Journal of Financial Economics, Vol. 68, No. 2, 2003, pp. 233-262. doi:10.1016/S0304-405X(03)00066-7

[11] Y. Li and W.-D. Zhang, "Theoretical Analysis on Combination Incentive to Operator," Journal of Industrial Engineering and Engineering Management, Vol. 16, No. 3, 2002, pp. 26-30.

[12] J.-T. He, D.-M. Zeng and Y.-H. Yao, "The Device of Incentive Combination to Top Management Team in China Listed Companies," Systems Engineering, Vol. 23, No. 12, 2005, pp. 44-47.

[13] J. Zhang, Z.-S. Zhang and L.-H. Zhao, "Study on Incentive Portfolio and Optimization of S\&T Professionals Based on Comprehensive Salary," Soft Science, Vol. 24, No. 12, 2010, pp. 27-33.

[14] G.-L. Liu, Y. Li and J. Li, "The Confirmation of the Incomplete Replacement between Material Motivation and Non-Material Motivation in Accounting Firm," Systems Engineering, Vol. 25. No. 2, 2007, pp. 124-126. 


\section{Appendix}

1) First-order results of Equation (8):

$$
\frac{\partial \alpha^{*}}{\partial \lambda}=\frac{\partial\left[\omega_{0}-\frac{n^{6}[1+(\varepsilon-\lambda) \gamma]^{2}\left(n^{3}-\rho b \sigma^{2}\right)}{2 b\left(n^{3}+\rho b \sigma^{2}\right)^{2}}\right]}{\partial \lambda}=\frac{n^{6}[1+(\varepsilon-\lambda) \gamma]^{2}\left(n^{3}-\rho b \sigma^{2}\right)}{2 b\left(n^{3}+\rho b \sigma^{2}\right)^{2}},
$$

since, the condition IR : $A C E \geq \omega_{0}$ holds, the following inequality holds, $n^{3}-\rho b \sigma^{2} \succ 0, \frac{\partial \alpha^{*}}{\partial \lambda} \succ 0$. so as the following inequality holds:

$$
\begin{gathered}
\frac{\partial \alpha^{*}}{\partial \varepsilon}=\frac{\partial\left[\omega_{0}-\frac{n^{6}[1+(\varepsilon-\lambda) \gamma]^{2}\left(n^{3}-\rho b \sigma^{2}\right)}{\left.2 b\left(n^{3}+\rho b \sigma^{2}\right)^{2}\right]}\right.}{\partial \varepsilon}=-\frac{\gamma n^{6}[1+(\varepsilon-\lambda) \gamma]\left(n^{3}-\rho b \sigma^{2}\right)}{b\left(n^{3}+\rho b \sigma^{2}\right)^{2}} \prec 0, \\
\frac{\partial \alpha^{*}}{\partial n}=\frac{\partial\left[\omega_{0}-\frac{n^{6}[1+(\varepsilon-\lambda) \gamma]^{2}\left(n^{3}-\rho b \sigma^{2}\right)}{\left.2 b\left(n^{3}+\rho b \sigma^{2}\right)^{2}\right]}\right.}{\partial n}=-\frac{3 n^{5}[1+(\varepsilon-\lambda) \gamma]^{2}\left[\left(n^{3}-\rho b \sigma^{2}\right)\left(n^{3}+\rho b \sigma^{2}\right)+2 \rho b \sigma^{2}\right]}{2 b\left(n^{3}+\rho b \sigma^{2}\right)^{3}} \prec 0, \\
\frac{\partial \alpha^{*}}{\partial \rho}=\frac{\partial\left[\omega_{0}-\frac{n^{6}[1+(\varepsilon-\lambda) \gamma]^{2}\left(n^{3}-\rho b \sigma^{2}\right)}{\left.2 b\left(n^{3}+\rho b \sigma^{2}\right)^{2}\right]}\right.}{\partial \rho}=\frac{\sigma^{2} n^{6}[1+(\varepsilon-\lambda) \gamma]^{2}\left(3 n^{3}-\rho b \sigma^{2}\right)}{2\left(n^{3}+\rho b \sigma^{2}\right)^{3}} \succ 0, \\
\frac{\partial \alpha^{*}}{\partial b}=\frac{\partial\left[\omega_{0}-\frac{n^{6}[1+(\varepsilon-\lambda) \gamma]^{2}\left(n^{3}-\rho b \sigma^{2}\right)}{2 b\left(n^{3}+\rho b \sigma^{2}\right)^{2}}\right]}{\partial b}=\frac{\rho \sigma^{2} n^{6}[1+(\varepsilon-\lambda) \gamma]^{2}\left(3 n^{3}-\rho b \sigma^{2}\right)}{2 b\left(n^{3}+\rho b \sigma^{2}\right)^{3}} \succ 0 .
\end{gathered}
$$

2) First-order results of Equation (9):

$$
\frac{\partial f\left(\alpha^{*}, \beta^{*}\right)}{\partial \lambda}=\frac{\partial\left[\frac{n^{6}[1+(\varepsilon-\lambda) \gamma]^{2}}{2 b\left(n^{3}+\rho b \sigma^{2}\right)}-\omega_{0}\right]}{\partial \lambda}=\frac{-\gamma n^{6}[1+\gamma(\varepsilon-\lambda)]}{b\left(n^{3}+\rho b \sigma^{2}\right)} \prec 0,
$$

since, the condition IR : $A C E \geq \omega_{0}$ holds, the following inequality holds, $n^{3}-\rho b \sigma^{2} \succ 0, \frac{\partial \alpha^{*}}{\partial \lambda} \succ 0$.

$$
\begin{gathered}
\frac{\partial f\left(\alpha^{*}, \beta^{*}\right)}{\partial \varepsilon}=\frac{\partial\left[\frac{n^{6}[1+(\varepsilon-\lambda) \gamma]^{2}}{2 b\left(n^{3}+\rho b \sigma^{2}\right)}-\omega_{0}\right]}{\partial \varepsilon}=\frac{\gamma n^{6}[1+\gamma(\varepsilon-\lambda)]}{b\left(n^{3}+\rho b \sigma^{2}\right)} \succ 0, \\
\frac{\partial f\left(\alpha^{*}, \beta^{*}\right)}{\partial n}=\frac{\partial\left[\frac{n^{6}[1+(\varepsilon-\lambda) \gamma]^{2}}{2 b\left(n^{3}+\rho b \sigma^{2}\right)}-\omega_{0}\right]}{\partial n}=\frac{3 n^{5}[1+(\varepsilon-\lambda) \gamma]^{2}\left(3 n^{3}+2 \rho b \sigma^{2}\right)}{2 b\left(n^{3}+\rho b \sigma^{2}\right)^{2}} \succ 0,
\end{gathered}
$$




$$
\begin{gathered}
\frac{\partial f\left(\alpha^{*}, \beta^{*}\right)}{\partial \rho}=\frac{\partial\left[\frac{n^{6}[1+(\varepsilon-\lambda) \gamma]^{2}}{2 b\left(n^{3}+\rho b \sigma^{2}\right)}-\omega_{0}\right]}{\partial \rho}=\frac{-n^{6}[1+(\varepsilon-\lambda) \gamma]^{2} \sigma^{2}}{2\left(n^{3}+\rho b \sigma^{2}\right)^{2}} \prec 0, \\
\frac{\partial f\left(\alpha^{*}, \beta^{*}\right)}{\partial b}=\frac{\partial\left[\frac{n^{6}[1+(\varepsilon-\lambda) \gamma]^{2}}{2 b\left(n^{3}+\rho b \sigma^{2}\right)}-\omega_{0}\right]}{\partial b}=\frac{-n^{6}[1+(\varepsilon-\lambda) \gamma]^{2}\left(n^{3}+2 \rho b \sigma^{2}\right)}{2 b^{2}\left(n^{3}+\rho b \sigma^{2}\right)^{2}} \prec 0
\end{gathered}
$$

